\title{
Characterisation of MHC class II DRB genes in the northern tree shrew (Tupaia belangeri)
}

\author{
Claus Oppelt · Rebecca Wutzler · Dietrich von Holst
}

\begin{abstract}
Genes of the major histocompatibility complex (MHC) mainly code for proteins of the immune system of jawed vertebrates. In particular, MHC class I and II cell surface proteins are crucial for the self/non-self discrimination of the adaptive immune system and are the most polymorphic genes in vertebrates. Positive selection, gene duplications and pseudogenes shape the face of the MHC and reflect a highly dynamic evolution. Here, we present for the first time data of the highly polymorphic MHC class II $D R B$ exon 2 of a representative of the mammalian order scandentia, the northern tree shrew Tupaia belangeri. We found up to eight different alleles per individual and determined haplotype constitution by intensively studying their inheritance. The alleles were assigned to four putative loci, all of which were polymorphic. Only the most polymorphic locus was subject to positive selection within the antigen binding sites and only alleles of this locus were transcribed.
\end{abstract}

C. Oppelt $\cdot$ R. Wutzler $\cdot$ D. von Holst

Department of Animal Physiology, University of Bayreuth, 95440 Bayreuth, Germany

Present Address:

C. Oppelt ( $₫)$

Limnological Institute, University of Konstanz,

Mainaustr. 252,

78464 Constance, Germany

e-mail: claus.oppelt@uni-konstanz.de

Present Address:

R. Wutzler

Department of Behavioural Ecology and Evolutionary Genetics,

Max Planck Institute for Ornithology,

82305 Seewiesen, Germany
Keywords Major histocompatibility complex . Peptide binding region - Polymorphism . Balancing selection · Positive selection $\cdot$ Tupaia

\section{Introduction}

The major histocompatibility complex of vertebrates contains over 100 genes predominantly expressing immunerelated proteins (The MHC sequencing consortium 1999; Kelley et al. 2005). In particular, the so-called classical MHC genes (class I and II genes) code for proteins that bind and present peptides on the surface of cells. T-cells recognise the combined structure of $\mathrm{MHC}$ protein and peptide, which finally triggers the adaptive immune response (Germain 1994).

The central role of classical MHC genes is supposed to be part of an evolutionary arms race between vertebrate hosts and their parasites, where parasites escape the recognition of their hosts' $\mathrm{MHC}$ genes. In turn the $\mathrm{MHC}$ repertoire of the hosts shifts due to natural and sexual selection (Apanius et al. 1997; Wegner et al. 2004). The highly polymorphic nature of MHC class I and II genes usually observed in wild populations is well explained by balancing selection (Apanius et al. 1997; Borghans et al. 2004; but see Stoffels and Spencer 2008; van Oosterhout 2009). This is acting through (1) an advantage for heterozygous individuals (overdominance) due to their wider antigen recognition spectrum (Doherty and Zinkernagel 1975), and (2) advantage to carriers of rare alleles because the adaptation of parasites will preferably target common alleles (Nei and Hughes 1991). Despite this diversifying selection, single alleles reveal a long natural history and survive even speciation events (trans-species polymorphism, Hughes and Yeager 1998). Furthermore, MHC diversity is 
increased by the duplication of loci (Parham and Ohta 1996) and recombination (Yeager and Hughes 1999).

However, MHC diversity within a single individual is finite, unlike antibody or T-cell receptor diversity. Thus, a too diverse MHC constitution could be detrimental due to an increased susceptibility to autoimmune reactions or increased negative thymic selection of T-cell clones (Nowak et al. 1992; but see Borghans et al. 2003). Hence, ongoing evolution with gene duplication might result in a loss of function of abundant genes in a birth and death process (Nei and Rooney 2005).

The role of MHC genes in host-parasite interaction and their highly polymorphic nature makes them interesting candidates for testing disease associations (e.g. Hill et al. 1997; Kettaneh et al. 2006; Westerdahl 2007) or hypotheses of sexual reproduction and mating strategies (Bernatchez and Landry 2003; Lampert et al. 2009). Therefore, MHC diversity has been examined in many vertebrate species. MHC diversity in mammals has been studied predominantly in primates and rodents focussing on the highly polymorphic MHC class II exon 2. But comparable data is still lacking from scandentia (tree shrews) and dermoptera (colugos), representing the closest living relatives of primates (Murphy et al. 2001; Janecka et al. 2007).

In the present study, we describe for the first time $\mathrm{MHC}$ DRB exon 2 diversity in a representative species of the order scandentia: the northem tree shrew (Tupaia belangeri). The squirrel-like scandentia (tree shrews) are exclusive to SouthEast Asia and contain two families: the ptilocercidae with only one species and the tupaiidae with 19 species (Olson et al. 2005), all of which are diurnal and omnivorous (Emmons 2000). The species of the genus Tupaia live in facultative monogamous social systems (Kawamichi and Kawamichi 1979; Emmons 2000; Oommen and Shanker 2008). The phylogenetic position of tree shrews was debated for many decades (Sargis 2004). Recent studies place them in a distinct order within the euarchontoglires (rodents, lagomorphs, primates, dermoptera, scandentia, Murphy et al. 2001), but their exact position is still unclear (see e.g. Murphy et al. 2001; Bininda-Emonds et al. 2007; Janecka et al. 2007).

We examined MHC class II DRB variation, haplotypic constitution of the DRB-region and gene transcription of individuals from a captive population with well-known genealogical structure.

\section{Material and methods}

Study animals

The tree shrews ( $T$. belangeri) originated from a breeding stock of the department of Animal Physiology at the University of Bayreuth and represent the up to 15th generation in captivity. The founders of the population were animals trapped at different sites in Thailand. Individuals were kept separately or as pairs, so that the ancestry of every individual was known. In total 230 tree shrews were genotyped for MHC DRB exon 2, whereas inheritance of most alleles was tracked over two or more generations.

\section{Major histocompatibility complex genotyping}

We extracted DNA using DNeasy Tissue Kit (Qiagen, Hilden) following the instructor's manual from tissue samples taken from the ear. We conducted polymerase chain reaction (PCR) using two primer systems: (1) Primer pair JS1 (GAG TGT CAT TTC TAC AAC GGG ACG) and JS2 (GAT CCC GTA GTT GTG TCT GCA; Schad et al. 2004). These amplify a $171 \mathrm{bp}$ fragment of the MHC class II DRB exon 2 containing most of the polymorphic peptide binding region. JSI/JS2 were originally designed based on prosimian sequences, but also perform successfully in rodents (Babik et al. 2005; Harf and Sommer 2005) and lagomorphs (Oppelt et al. 2010). The primer pair GH46 (CCG GAT CCT TCG TGT CCC CAC AGC ACG) and GH50 (CTC CCC AAC CCC GTA GTT GTG TCT GCA; Erlich and Bugawan 1990) target all DRB genes of the human MHC, but amplify only a subset in T. belangeri. We accomplished PCR in $20 \mu \mathrm{l}$ reaction volumes with $1 \mu \mathrm{l}$ DNA, $1.5 \mathrm{mM} \mathrm{MgCl}_{2}, 0.28 \mu \mathrm{M}$ of each primer (MWG Biotech, Ebersberg), $140 \mu \mathrm{M}$ of each dNTP (Roth, Karlsruhe) and $0.5 \mathrm{U}$ polymerase (Biotherm Polymerase, Genecraft, Köln). Cycling conditions for primer system JS1/JS2 were: $5 \mathrm{~min}$ at $94^{\circ} \mathrm{C}, 30$ cycles of $1 \mathrm{~min}$ at $94^{\circ} \mathrm{C}$, $1 \mathrm{~min}$ at $55^{\circ} \mathrm{C}, 1 \mathrm{~min}$ at $72^{\circ} \mathrm{C}$ and a final elongation step of $7 \mathrm{~min}$ at $72^{\circ} \mathrm{C}$. Cycling conditions for primer system $\mathrm{GH} 46 /$ GH50 were: $5 \mathrm{~min}$ at $94^{\circ} \mathrm{C}, 35$ cycles of $1 \mathrm{~min}$ at $94^{\circ} \mathrm{C}$, $1 \mathrm{~min}$ at $61^{\circ} \mathrm{C}, 1 \mathrm{~min}$ at $72^{\circ} \mathrm{C}$ and a final elongation of $7 \mathrm{~min}$ at $72^{\circ} \mathrm{C}$.

For DRB genotyping we conducted a combined single strand conformation polymorphism/heteroduplex analysis (SSCP/HD; according to Fain et al. 2001). We mixed $5 \mu$ l of each PCR product with an equal volume of $95 \%$ formamide, denatured for $5 \mathrm{~min}$ at $96^{\circ} \mathrm{C}$ and immediately chilled on ice water for $10 \mathrm{~min}$. The mixture was loaded on a $9 \%$ nondenaturing polyacrylamide gel $(37.5: 1)$. Electrophoresis was carried out at $10^{\circ} \mathrm{C}$ for $3 \mathrm{~h}$ with $8 \mathrm{~W}$ per gel. Under these conditions, denatured DNA forms double-stranded heteroduplices (HD) and single-stranded conformations (SSCP). Both are sequence dependent and thus allow discriminating different genotypes. Electrophoresis was followed by a sensitive silver-staining protocol (Budowle et al. 1991). Samples with equal banding patterns were rearranged and run again on a non-denaturing PAGE on adjacent lanes to ensure the genotyping. Individuals with unique genotypes were typed using two independent PCR reactions. If 
possible, we cut all SSCP-bands from at least two individuals of each genotype and incubated them in $80 \mu \mathrm{l}$ water for at least $3 \mathrm{~h}$ at $37^{\circ} \mathrm{C}$. One microlitre was used as template in another PCR under the conditions described above. We screened the products in an additional SSCP analysis and every allele was sequenced in both directions (Seqlab, Göttingen) from at least two individuals or two independent PCRs from one individual.

\section{Haplotype inference}

A haplotype is defined as the set of allelic variants that are inherited as a unit. The close proximity of DRB loci in mammals leads to an inheritance in linkage groups. This makes direct observation of recombination very improbable, but simplifies the identification of haplotypes. According to Mendelian rules, the two parental genotypes form up to four different genotypes in the progeny. Those alleles, which segregate as units from the parents to the offspring can therefore be identified as haplotypes. Most haplotypes could be inferred in this way. Anyway, some individuals from earlier generations possessed unique genotypes, but we lacked genetic samples from direct kin.

\section{Transcription analysis}

In order to investigate allele specific transcription, we analysed DRB sequences on the CDNA level of 8 tree shrews with already known genotype. Animals were sacrificed, spleen tissue removed and stored in RNAlater (Qiagen) until analysis. We extracted RNA (total RNA kit, Peqlab) and removed genomic DNA by incubation of $1 \mu$ of RNA solution with $1 \mathrm{U}$ DNAse (Fermentas) in $10 \mu \mathrm{l}$ volumes for $30 \mathrm{~min}$ at $37^{\circ} \mathrm{C}$. We performed a cDNA synthesis with reverse transcriptase and oligo(dT)18-primer (first strand cDNA synthesis kit, Fermentas) in $20 \mu \mathrm{l}$ reaction volumes with incubation at $55^{\circ} \mathrm{C}$ for $45 \mathrm{~min}$ and inactivation at $70^{\circ} \mathrm{C}$ for $15 \mathrm{~min}$. We used the obtained cDNA as a template for PCR with the primer system JS1/JS2 under the conditions described above. DNAse digested RNA solutions served as negative controls to ensure the full elimination of genomic DNA. The amplicons of cDNA were loaded on a nondenaturing gel for SSCP/HD analysis; bands were cut out, reamplified and sequenced. The procedure was not applicable with primer pair $\mathrm{GH} 46 / \mathrm{GH} 50$, because the forward primer GH46 spans the intron/exon border.

Sequence analysis procedure and statistical analyses

Sequences were aligned manually with Bioedit 7.09 (Hall 1999). We truncated longer fragments obtained by the primer system $\mathrm{GH} 46 / \mathrm{GH} 50$, to fit the $171 \mathrm{bp}$ fragment of primer system JS1/JS2 and conducted a Blast (megablast) search (Altschul et al. 1990) to find the most homologous sequences available.

We constructed a neighbour joining tree in Mega 4.1 (Tamura et al. 2007) with 5,000 bootstraps to identify clusters potentially representing loci. Due to sequence similarity of some Tube-DRB alleles with class II sequences of laurasiatheria, we chose an afrotherian class II allele as outgroup (whole genome shotgun sequence from African elephant Loxodonta africana; accession number: AAGU03092621). The proportion of synonymous (dS) and non-synonymous (dN) substitutions was calculated for the 15 putative antigen binding sites defined by Brown et al. (1993) and the remaining non-antigen binding sites. We used the Z-Test implemented in Mega 4.1 to test for directed selection. This was done for all sequences in a combined analysis and for each of the four putative loci.

We tested gene conversion using Genecov 1.81 (Sawyer 1999) including all 29 alleles. Since DRB1 and DRB2 sequences form a cluster in a neighbour joining tree (Fig. 2, cluster I), we analysed for possible breakpoints within the alleles of these two putative loci. This was done with the computer programme GARD (Kosakovsky Pond et al. 2006) provided in the datamonkey web server (Kosakovsky Pond and Frost 2005).

\section{Results}

\section{MHC variability}

In 230 tree shrews typed for MHC DRB exon 2 with two primer systems we identified 29 alleles (Fig. 1), named according to the nomenclature of Klein et al. (1990) Tube $D R B^{*} 01$ to Tube $D R B^{*} 29$ (Accession numbers GU825729-GU825757). Each primer system amplified a subset of the total alleles with a certain overlap of alleles that were achieved with both primer systems. The majority of alleles were obtained by the primer system JS1/JS2. Only three alleles were exclusively amplified by GH46/ GH50 (*23,*25 and *27). An extensive Blast search with all identified sequences affirmed the homology to DRB sequences. Tube-DRB1 sequences were most homologous to primate DRB sequences, while Tube-DRB2, DRB3 and DRB4 alleles were homologous to primate and rodent DRB sequences as well as class II genes of some laurasiatheria (mainly ruminant DRB and cetacean DQB, Table 2, Electronic supplementary material). The pair wise distance between all Tube-DRB alleles ranged between 1 and 48 of the $171 \mathrm{bp}$ (mean, $29.05 \mathrm{bp} \pm 2.73 \mathrm{SE}$; bootstrap with 500 iterations). One allele (Tube $D R B^{*} 2 l$ ) had a triplet deletion on the last position, so that the amplicon was only $168 \mathrm{bp}$ in length. Tube $D R B^{*} 27$ had a stop codon within the sequence; 88 of the 171 positions were polymorphic. 


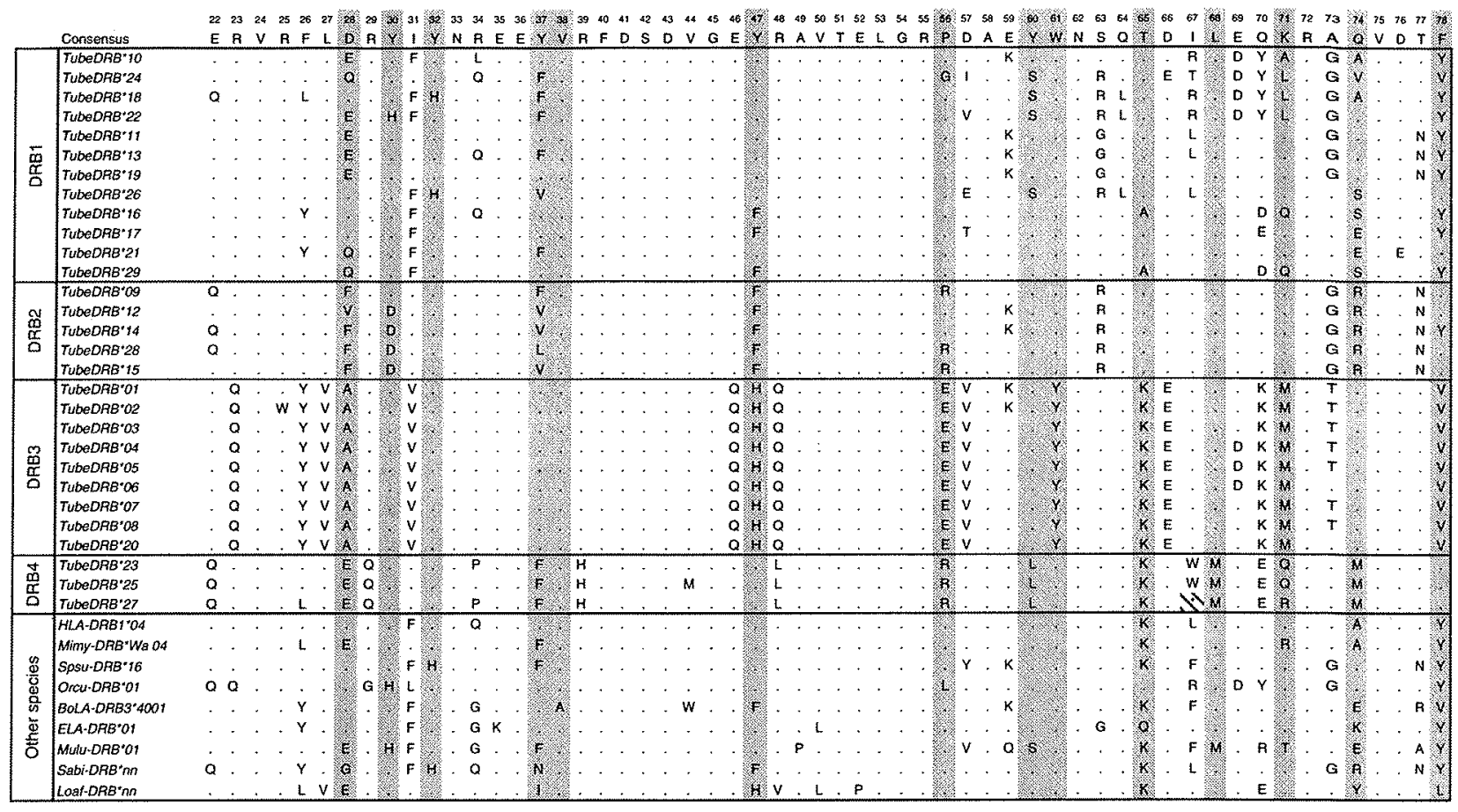

Fig. 1 Amino acid sequences of the 29 identified DRB alleles of Tupaia belangeri. A dot indicates a residue identical to the consensus sequence. The sites of the human putative peptide binding region according to Brown et al. (1993) are shaded grey, DRB1-DRB4 indicate the affiliation to the four putative DRB loci in the tree shrew. For reference, several other mammalian DRB sequences are listed:
HLA human, Mimy pygmy mouse lemur, Spsu spotted souslik, Orcu European rabbit, BoLA zebu cattle, ELA horse, Mulu European mink, $S a b i$ greater sac-winged bat, Loaf African elephant. Accession numbers: EF495129; AB078291; EF569201; GQ168795; 282025; L25644; EU263550; EF533891; AAGU03092621

ratio of non-synonymous to synonymous substitutions in antigen binding sites $(\mathrm{ABS})$ and non-antigen binding sites (non-ABS) according to Brown et al. (1993) using all alleles and alleles of the four putative loci separately. We found significant signs of positive selection with a $\mathrm{dN} / \mathrm{dS}$ ratio of 4.13 only in the ABS of putative DRBl alleles ( $Z$ test, $p<$ $0.05)$ and within all alleles with a $\mathrm{dN} / \mathrm{dS}$ ratio of $2.04(p<0.01$, Table 1). DRB1 is the most polymorphic locus and a representing allele was found in every individual. In contrast, overall non-ABS were subject to purifying selection (Table 1).

\section{Transcription}

Eight tree shrews with known MHC DRB genotypes were investigated for transcription of alleles. These animals covered the haplotypes $\# 2, \# 3, \# 4, \# 5, \# 6, \# 8, \# 9$ and $\# 10$ (see Fig. 4). All alleles that were found to be transcribed in these eight tree shrews belonged to the putative locus DRB1. All DRB2 and DRB3 alleles present on the respective haplotypes were not transcribed (Fig. 2). DRB4 transcription could not be tested because the respective alleles only were amplified by the primer system $\mathrm{GH} 46 / \mathrm{GH} 50$, which is partly intronic. But at least one of the three DRB4 alleles represents a pseudoallele comprising a stop codon.
Functional MHC genes show signs of positive selection on antigen binding sites (e.g. Axtner and Sommer 2007; Hauswaldt et al. 2007; Archie et al. 2010). We analysed the not have DNA from related individuals to obtain these haplotypes in different genotypic combinations.

\section{Positive selection}


Fig. 2 Neighbour-joining tree of the 29 Tube-DRB alleles. An African elephant MHC class II sequence (AAGU03092621) served as outgroup. Numbers indicate bootstrap support $\geq 50$. Black dot transcribed allele; grey dot allele is not transcribed; white dot pseudoallele

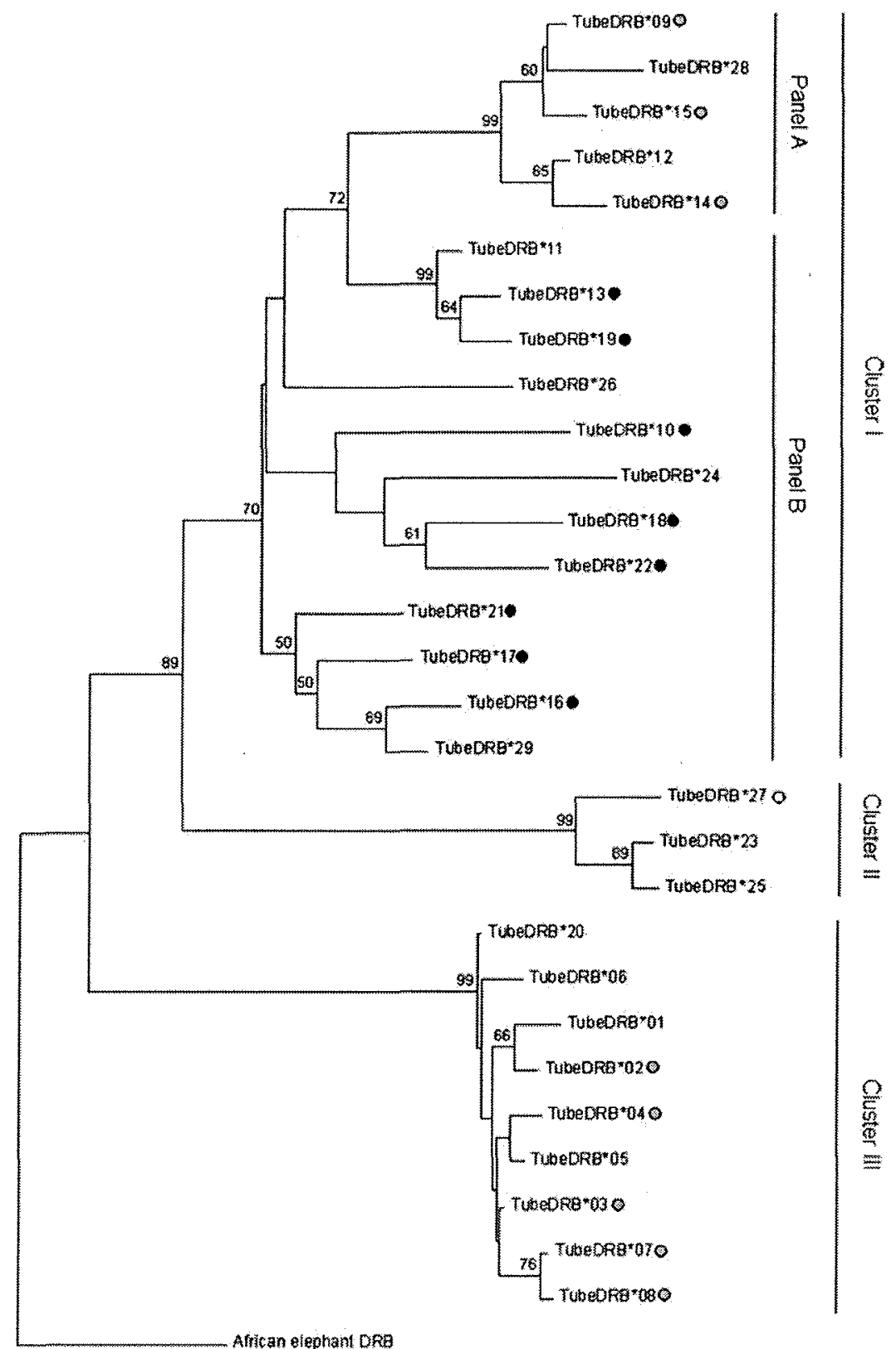

Recombination

The constitution of haplotypes reveals that the alleles DRB*03, *04 and *13 appear each on two different haplotypes, indicating interlocus recombination in the past. Furthermore, analysis of gene conversion revealed a recombination between Tube $D R B^{*} 18$ and Tube $D R B^{*} 21$. Finally, we identified a significant breakpoint at position 104 within the DRB1 and DRB2 sequences.

\section{Discussion}

In the present study we characterised MHC class II DRB genes of a representative of the order scandentia, the tree shrew $T$. belangeri. We found 14 haplotypes with up to four copies of DRB genes but only one of them was transcribed. This locus was the most polymorphic and proved to be under strong positive selection within the peptide binding region. 


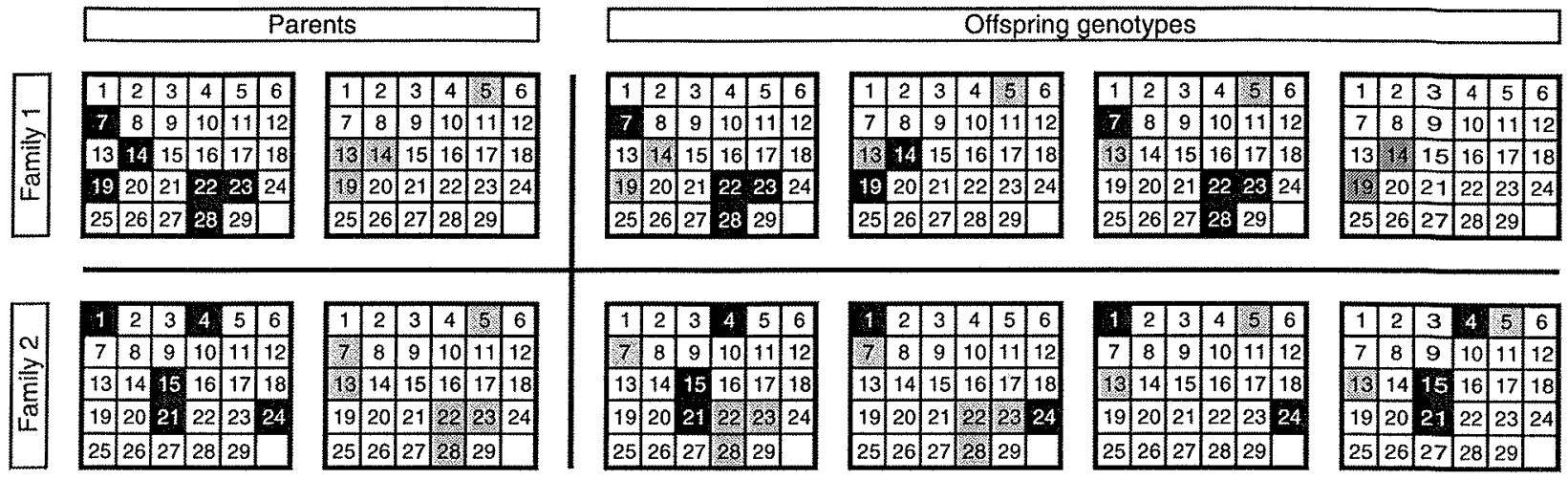

Fig. 3 Two examples of haplotype inference by segregation of alleles within families. The two boxes on the left of each row represent the genotypes of a breeding pair and the four right boxes represent the four

\section{Composition of $T$. belangeri DRB haplotypes}

Individual tree shrews carried two to eight different DRB alleles based on 14 haplotypes composed of one to four alleles. This number is high in comparison to what was found in several other mammalian species, where only one or two identified offspring genotypes of that pair. Numbers in squares are in correspondence with allele numbering (Tube-DRB*01-29)

loci were identified (e.g. white-tailed deer, Van Den Bussche et al. 1999; Eurasian beaver, Babik et al. 2005; hairy-footed gerbil, Harf and Sommer 2005; European mink, Becker et al. 2009). However, in these species as well as in tree shrews the observed number of MHC genes is probably an underestimation of the real number of DRB genes, because further
Fig. 4 Constitution of 14 DRB haplotypes in the northem tree shrew mostly inferred from linked inheritance. The numbers in brackets give the number of haplotypes found in the breeding stock. Haplotypes \#12 and \#14 could not be sequenced completely due to SSCP banding pattern complexity and low frequency. Therefore, the position of a box with a question mark symbol is determined by similarity of partial sequences to other tree shrew DRB alleles

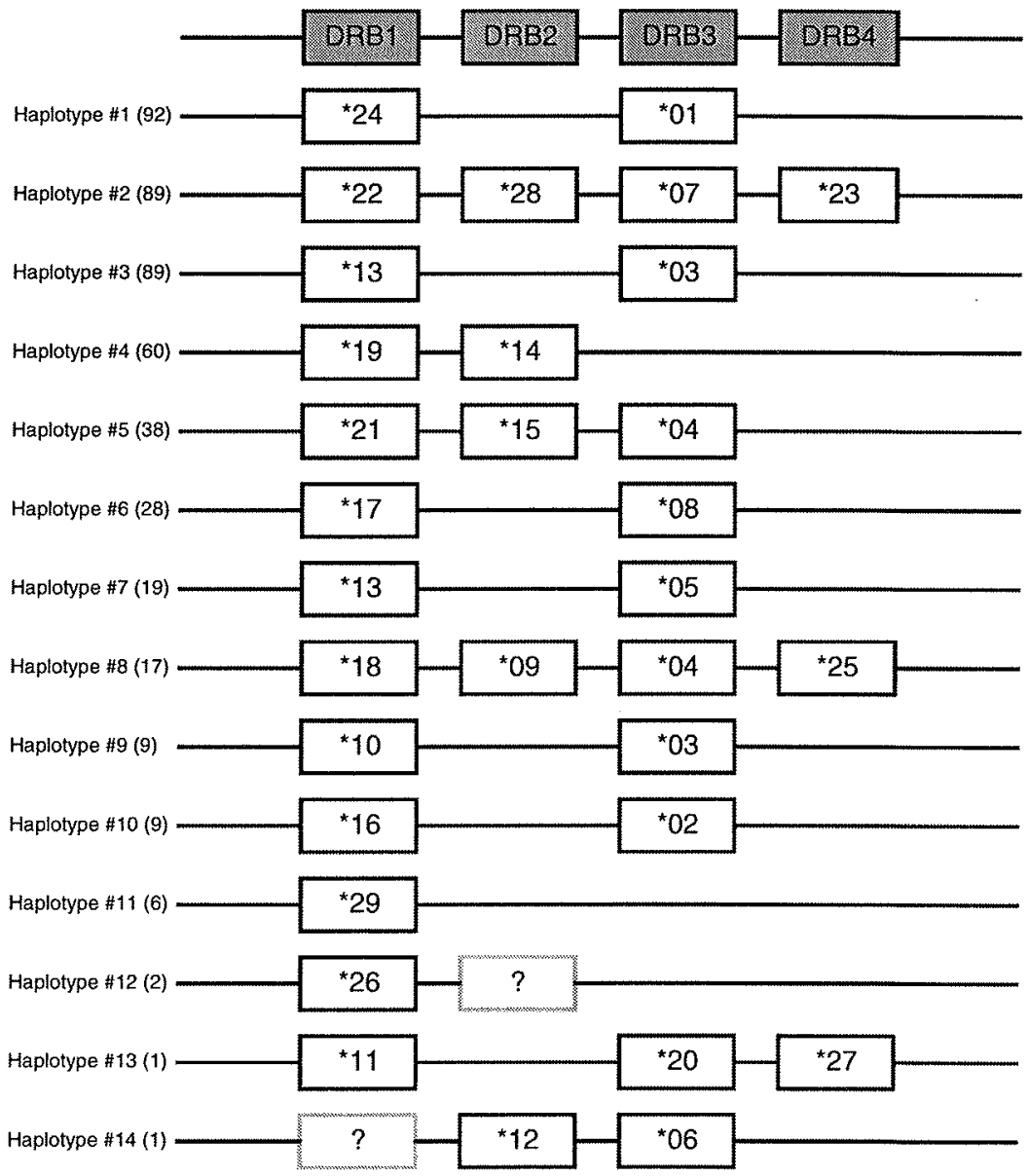


Table 1 The ratio of nonsynonymous to synonymous substitutions $(\mathrm{dN} / \mathrm{dS})$ at putative antigen binding sites (ABS) according to Brown et al. (1993) and the remaining DRB exon 2 (non-ABS)

The Z-test for directed selection implemented in Mega 4.1 (Tamura et al. 2007) was conducted on all 29 alleles together and separately on the alleles of the four putative loci

\begin{tabular}{lllllr}
\hline & $\mathrm{dN}$ & $\mathrm{dS}$ & $\mathrm{dN} / \mathrm{dS}$ & $\mathrm{p}$ & $\mathrm{Z}$ \\
\hline ABS $N=15$ & & & & \\
DRB1 & $0.252 \pm 0.051$ & $0.061 \pm 0.051$ & $\mathbf{4 . 1 3 1}$ & $\mathbf{0 . 0 1 3}$ & 2.508 \\
DRB2 & $0.078 \pm 0.031$ & $0.159 \pm 0.069$ & 0.491 & 0.243 & -1.174 \\
DRB3 & $0.000 \pm 0.0$ & $0.056 \pm 0.038$ & 0.000 & 0.129 & -1.527 \\
DRB4 & $0.018 \pm 0.018$ & $0.075 \pm 0.085$ & 0.240 & 0.516 & -0.652 \\
All alleles & $0.345 \pm 0.042$ & $0.169 \pm 0.049$ & $\mathbf{2 . 0 4 1}$ & $\mathbf{0 . 0 0 5}$ & 2.828 \\
Non-ABS $N=42$ & & & & & -0.169 \\
DRB1 & $0.074 \pm 0.019$ & $0.080 \pm 0.022$ & 0.925 & 0.866 & -1.216 \\
DRB2 & $0.013 \pm 0.009$ & $0.045 \pm 0.025$ & 0.289 & 0.226 & -1.298 \\
DRB3 & $0.016 \pm 0.008$ & $0.045 \pm 0.022$ & 0.356 & 0.197 & -0.721 \\
DRB4 & $0.021 \pm 0.012$ & $0.046 \pm 0.031$ & 0.457 & 0.472 & -2.123 \\
All alleles & $0.095 \pm 0.018$ & $0.186 \pm 0.036$ & $\mathbf{0 . 5 1 1}$ & $\mathbf{0 . 0 3 6}$ & \\
\hline
\end{tabular}

genes may have failed to be amplified by the primer systems used. Thus, some species that are studied in more detail also show more loci (e.g. European rabbit, Sittisombut et al. 1989; humans, Trowsdale 1995; sheep, Dukkipati et al. 2006).

Also the different number of genes on the different tree shrew DRB haplotypes may be due to a real copy number variation or primer mismatches leading to null alleles. Although we cannot rule out the latter case, DRB gene copy number variation seems to be common in many fish (Nile tilapia, Malaga-Trillo et al. 1998; threespined stickleback, Sato et al. 1998; Eurasian perch, Michel et al. 2009), but also in mammals (e.g. cattle, Ellis and Ballingall 1999; primates, Bontrop 2006; bank vole, Kloch et al. 2010).

Based on the allelic composition of the haplotypes, we hypothetically assigned individual alleles to the four loci, assuming that alleles of a certain MHC locus tend to form a phylogenetic cluster. In fact this has been observed several times in MHC class II exon 2 (Figueroa et al. 1994; Andersson 1998; Lukas et al. 2004). On the other hand, if an allele undergoes duplication, the alleles of the second gene will be closely related to the original allele of the first gene, confusing a strict phylogenetic clustering of the loci.

The topology of tree shrew DRB sequences reveals two clusters (clusters II and III, Fig. 2) to be strikingly distant to the majority of alleles. Both cluster II and cluster III alleles perfectly behave like true alleles, i.e. we never found more than one allele per cluster in a haplotype nor more that two in one individual.

In contrast, we found several haplotypes harbouring two alleles out of the highly divergent cluster $\mathrm{I}$, which therefore represents two loci. In those cases, one allele always originated from panel $A$ and the other from panel $B$ (e.g. $D R B^{*} 14$ and $* 19$, Fig. 2). This indicates that the panels $A$ and $B$ form distinct loci, although they do not mirror phylogenetic relationships. This could be for at least two reasons: firstly, the alleles found in panel $\mathrm{A}\left(D R B^{*} 09\right.$, $* 12, * 14, * 15$ and $* 28$ ) could result from a duplication of an allele closely related to $D R B^{*} 11, * 13$ and $* 19$. Therefore, these eight alleles could cluster, although they belong to different loci. Secondly, sequence motives could be mixed up by past asymmetric recombinations between the two loci. In fact we found one putative breakpoint within the alleles of these two loci. A separate analysis of the two fragments revealed different topologies, whilst the neighbour joining tree of the first fragment showed a phylogenetic clustering according to the four previously defined putative loci (Fig. 5, Electronic supplementary material). The strongest indication of the locus nature of the panels $A$ and $B$ was provided by the transcription experiment, where we proved eight of 12 panel-B-alleles to be transcribed and three of five panel-A-alleles to be not. The most likely scenario for this observation is that the two panels correspond to two loci (DRB1 and DRB2), of which only one is transcribed. Panel B therefore represents the most diverse and the only transcribed locus (DRB1) in $T$. belangeri. Although the assignment of alleles to different loci might be most likely, it remains hypothetical.

\section{Functional polymorphism of tree shrew DRB genes}

Classical MHC genes represent the most polymorphic genes in vertebrates (Apanius et al. 1997). Antigen binding sites of functional MHC genes underlie positive selection, and therefore show an exceeding rate of non-synonymous over synonymous substitutions (Bernatchez and Landry 2003; Sommer 2005). Futhermore, MHC polymorphism is also maintained by gene conversion and gene duplication (Yeager and Hughes 1999). However, exaggerated MHC diversity may have disadvantages for the individual by increasing the possibility of autoimmune reactions or magnifying negative T-cell selection in the thymus (Nowak et al. 1992). Therefore there might be a selective advantage of a functional loss of abundant DRB loci in a birth and death process (Sawai et al. 2008). 
Our data on tree shrew DRB sequences were in accordance with all of these assumptions. Evidence was given by significant positive selection within the peptide binding region considering all tree shrew DRB sequences $(\mathrm{dN} / \mathrm{dS}=2.04)$. However, a more detailed exploration of the single putative loci revealed that this effect was due to DRB 1 alleles only, showing an even higher $\mathrm{dN} / \mathrm{dS}$ ratio $(>4)$. In contrast, the other three loci show no signs of directed selection. This result fits well with the transcription pattern. We recovered only DRBI sequences on the CDNA level. DRB2 and DRB3 sequences were not transcribed and at least one of the three DRB4 sequences appears to be a pseudoallele due to a stop codon.

Since we only obtained partial sequences, it remains possible that the complete genes DRB2 to DRB4 represent pseudogenes, comparable to some DRB loci in humans (Horton et al. 2004). On the other hand, DRB2 to DRB4 could potentially be transcribed and expressed at very low levels, thus we were not able to detect transcription. Such low expression levels are e.g. known for the human gene DRB6 (Fernandez-Soria et al. 1998), but its functional significance is unknown.

We found only weak support for recombination within tree shrew DRB haplotypes and alleles. E.g. both alleles $D R B^{*} 03$ and $D R B^{*} 13$ together form a haplotype, but also appear in other combinations, indicating interlocus recombination. Gene conversion analysis of all alleles revealed only one internal recombination between two DRB 1 alleles. In contrast to other species studied in this context (e.g. deer mouse, Richman et al. 2003; three-spined stickleback, Reusch and Langefors 2005; chamois, Schaschl et al. 2005; Eurasian perch, Michel et al. 2009), recombination might play only a secondary role in tree shrews, but nevertheless contributes to sequence diversity.

\section{Homology of tree shrew DRB alleles}

The 29 tree shrew DRB sequences cluster in highly divergent lineages. None of the tree shrew alleles was found in other species, when analysed in a systematic Blast search. Tube-DRB1 sequences were found as most similar to primate $\mathrm{DRB}$ sequences, indicating relatively close phylogenetic relationship of the DRB1 locus to primate DRB genes. Instead, the best Blast hits of Tube-DRB2, 3 and 4 alleles were homologous to DRB sequences of primates, rodents, ruminants, carnivores and DQB alleles of whales. Especially DRB3 and DRB4 alleles were generally more distant to class II sequences published in Genbank so far.

DRB genes of primates are thought to share monophyletic ancestry in a single locus; therefore, duplications of primate DRB genes have been emerged after the separation of tree shrew and primate ancestors (Figueroa et al. 1994;
Kupfermann et al. 1999). Due to the sequence similarity the DRB1 locus of tree shrews might be orthologous to the ancestral primate DRB gene. In contrast DRB2, 3 and 4 alleles could have evolved independently in tree shrews or might be more related to DRB genes that have diverged in other taxa, but have been deleted in primates.

\section{Conclusion}

This study presents data on the MHC DRB region of the northern tree shrew ( $T$. belangeri). We provide evidence for the existence of at least four DRB loci in this species, all of which are polymorphic, but only one is transcribed. In general, we found only few indications for recombination. The transcribed locus DRBI is highly divergent and subject to positive selection within the peptide binding region. The other three loci DRB2 to DRB4 are also polymorphic, but probably not transcribed and selection is consistently neutral.

\section{References}

Altschul SF, Gish W, Miller W, Myers EW, Lipman DJ (1990) Basic local alignment search tool. J Mol Biol 215:403-410

Andersson G (1998) Evolution of the human HLA-DR region. Front Biosci 3:d739-d745

Apanius V, Penn D, Slev PR, Ruff LR, Potts WK (1997) The nature of selection on the major histocompatibility complex. Crit Rev Immunol 17:179-224

Archie EA, Henry T, Maldonado JE, Moss CJ, Poole JH, Pearson VR, Murray S, Alberts SC, Fleischer RC (2010) Major histocompatibility complex variation and evolution at a single, expressed DQA locus in two genera of elephants. Immunogenetics 62:85100

Axtner J, Sommer S (2007) Gene duplication, allelic diversity, selection processes and adaptive value of MHC class II DRB genes of the bank vole, Clethrionomys glareolus. Immunogenetics 59:417-426

Babik W, Durka W, Radwan J (2005) Sequence diversity of the MHC DRB gene in the Eurasian beaver (Castor fiber). Mol Ecol 14:4249-4257

Becker L, Nieberg C, Jahreis K, Peters E (2009) MHC class II variation in the endangered European mink Mustela lutreola (L. 1761)-consequences for species conservation. Immunogenetics $61: 281-288$

Bernatchez L, Landry C (2003) MHC studies in nonmodel vertebrates: what have we learned about natural selection in 15 years? $\mathrm{J}$ Evol Biol 16:363-377

Bininda-Emonds ORP, Cardillo $M$, Jones KE, MacPhee RDE, Beck RMD, Grenyer R, Price SA, Vos RA, Gittleman JL, Purvis A (2007) The delayed rise of present-day mammals. Nature 446:507-512

Bontrop RE (2006) Comparative genetics of MHC polymorphisms in different primate species: duplications and deletions. Hum Immunol 67:388-397

Borghans JAM, Noest AJ, De Boer RJ (2003) Thymic selection does not limit the individual MHC diversity. Eur J Immunol 33:33533358 
Borghans JAM, Beltman JB, Boer RJ (2004) MHC polymorphism under host-pathogen coevolution. Immunogenetics 55:732-739

Brown JH, Jardetzky TS, Gorga JC, Stern LJ, Urban RG, Strominger JL, Wiley DC (1993) Three-dimensional structure of the human class II histocompatibility antigen HLA-DR1. Nature 364:33-39

Budowle B, Chakraborty R, Giusti AM, Eisenberg AJ, Allen RC (1991) Analysis of the VNTR locus D1S80 by the PCR followed by high-resolution PAGE. Am J Hum Genet 48:137-144

Doherty PC, Zinkernagel RM (1975) A biological role for the major histocompatibility antigens. Lancet 1:1406-1409

Dukkipati VSR, Blair HT, Garrick DJ, Murray A, North P, Zealand N (2006) 'Ovar-Mhc'-ovine major histocompatibility complex: structure and gene polymorphisms. Genet Mol Res 5:581-608

Ellis SA, Ballingall KT (1999) Cattle MHC: evolution in action? Immunol Rev 167:159-168

Emmons L (2000) Tupai: a field study of Bornean treeshrews University of California Press, Berkeley

Erlich HA, Bugawan TL (1990) HLA DNA typing. In: Innis MA Gelfand DH, Sninsky JJ, White TJ (eds) PCR protocols: a guide to methods and applications. Academic Press, New York, pp 261-271

Fain MA, Zhao T, Kindt TJ (2001) Improved typing procedure for the polymorphic single-copy RLA-DQA gene of the rabbit reveals a new allele. Tissue Antigens 57:332-338

Fernandez-Soria VM, Morales P, Castro MJ, Suarez B, Recio MJ, Moreno MA, Paz-Artal E, Arnaiz-Villena A (1998) Transcription and weak expression of HLA-DRB6: a gene with anomalies in exon 1 and other regions. Immunogenetics 48:16-21

Figueroa F, O'hUigin C, Tichy H, Klein J (1994) The origin of the primate Mhc-DRB genes and allelic lineages as deduced from the study of prosimians. J Immunol 152:4455-4465

Germain RN (1994) MHC-dependent antigen processing and peptide presentation: providing ligands for $\mathrm{T}$ lymphocyte activation. Cell 76:287-299

Hall TA (1999) BioEdit: a user-friendly biological sequence alignment editor and analysis program for Windows $95 / 98 / \mathrm{NT}$. Nucleic Acids Symp Ser 41:95-98

Harf R, Sommer S (2005) Association between major histocompatibility complex class II DRB alleles and parasite load in the hairyfooted gerbil, Gerbillurus paeba, in the southern Kalahari. Mo Ecol 14:85-91

Hauswaldt JS, Stuckas H, Pfautsch S, Tiedemann R (2007) Molecular characterization of $\mathrm{MHC}$ class II in a nonmodel anuran species, the fire-bellied toad Bombina bombina. Immunogenetics 59:479-491

Hill AVS, Jepson A, Plebanski M, Gilbert SC (1997) Genetic analysis of host-parasite coevolution in human malaria. Philosophical Transactions of the Royal Society B: Biological Sciences $352: 1317-1325$

Horton R, Wilming L, Rand V, Lovering RC, Bruford EA, Khodiyar VK, Lush MJ, Povey S, Talbot CC, Wright MW (2004) Gene map of the extended human MHC. Nat Rev Genet 5:889-899

Hughes AL, Yeager M (1998) Natural selection at major histocompatibility complex loci of vertebrates. Annu Rev Genet 32:415-435

Janecka JE, Miller W, Pringle TH, Wiens F, Zitzmann A, Helgen KM, Springer MS, Murphy WJ (2007) Molecular and genomic data identify the closest living relative of primates. Science 318:792-794

Kawamichi T, Kawamichi M (1979) Spatial organization and territory of three shrews (Tupaia glis). Anim Behav 27:381-393

Kelley J, Walter L, Trowsdale $\mathbf{J}$ (2005) Comparative genomics of major histocompatibility complexes. Immunogenetics 56:683-695

Kettaneh A, Seng L, Tiev K, Toledano C, Fabre B, Cabane J (2006) Human leukocyte antigens and susceptibility to tuberculosis: a meta-analysis of case-control studies. Int $\mathrm{J}$ Tuberc Lung Dis 10:717--725

Klein J, Bontrop RE, Dawkins RL, Erlich HA, Gyllensten UB, Heise ER, Jones PP, Parham P, Wakeland EK, Watkins DI (1990)
Nomenclature for the major histocompatibility complexes of different species: a proposal. Immunogenetics 31:217-219

Kloch A, Babik W, Bajer A, Sinski E, Radwan J (2010) Effects of an MHC-DRB genotype and allele number on the load of gut parasites in the bank vole Myodes glareolus. Mol Ecol 19:255-265

Kosakovsky Pond SL, Frost SDW (2005) DATAMONKEY: rapid detection of selective pressure on individual sites of codon alignments. Bioinformatics 21:2531-2533

Kosakovsky Pond SL, Posada D, Gravenor MB, Woelk CH, Frost SDW (2006) Automated phylogenetic detection of recombination using a genetic algorithm. Mol Biol Evol 23:1891-1901

Kupfermann H, Satta Y, Takahata N, Tichy H, Klein J (1999) Evolution of Mhc-DRB introns: implications for the origin of primates. J Mol Evol 48:663-674

Lampert KP, Fischer P, Schartl M (2009) Major histocompatibility complex variability in the clonal Amazon molly, Poecilia formosa: is copy number less important than genotype? Mol Ecol 18:1124-1136

Lukas D, Bradley BJ, Nsubuga AM, Doran-Sheehy D, Robbins MM, Vigilant L (2004) Major histocompatibility complex and microsatellite variation in two populations of wild gorillas. Mol Ecol $13: 3389-3402$

Malaga-Trillo E, Zaleska-Rutczynska Z, McAndrew B, Vincek V, Figueroa F, Sultmann $H$, Klein J (1998) Linkage relationships and haplotype polymorphism among cichlid Mhc class II B loci. Genetics 149:1527-1537

Michel C, Bernatchez L, Behrmann-Godel J (2009) Diversity and evolution of MHII $\beta$ genes in a non-model percid species-the Eurasian perch (Perca fluviatilis L). Mol Immunol 46:3399-3410

Murphy WJ, Eizirik E, O'Brien SJ, Madsen O, Scally M, Douady CJ, Teeling E, Ryder OA, Stanhope MJ, de Jong WW (2001) Resolution of the early placental mammal radiation using Bayesian phylogenetics. Science 294:2348-2351

Nei M, Hughes AL (1991) Polymorphism and evolution of the major histocompatibility complex loci in mammals. In: Evolution at the molecular level. Sinauer Associates Inc., Sunderland, pp 222 247

Nei M, Rooney A (2005) Concerted and birth-and-death evolution of multigene families. Annu Rev Genet 39:121-152

Nowak MA, Tarczy-Homoch K, Austyn JM (1992) The optimal number of major histocompatibility complex molecules in an individual. Proceedings of the National Academy of Sciences 89:10896-10899

Olson L, Sargis E, Martin R (2005) Intraordinal phylogenetics of treeshrews (Mammalia: Scandentia) based on evidence from the mitochondrial 12S rRNA gene. Mol Phylogenet Evol 35:656673

Oommen MA, Shanker K (2008) Ecology and bahaviour of an endemic treeshrew Tupaia nicobarica Zelebor 1869 on great nicobar island. India $J$ Bombay Nat Hist Soc 105:55-63

Oppelt C, Starkloff A, Rausch P, von Holst D, Rödel HG (2010) Variation of major histocompatibility complex and age-specific endoparasite load in subadult European rabbits. Molecular Ecology (in press)

Parhan P, Ohta T (1996) Population biology of antigen presentation by MHC class I molecules. Science 272:67-74

Reusch TBH, Langefors $\AA$ (2005) Inter-and intralocus recombination drive MHC class IIB gene diversification in a teleost, the threespined stickleback Gasterosteus aculeatus. J Mol Evol 61:531541

Richman AD, Herrera LG, Nash D, Schierup MH (2003) Relative roles of mutation and recombination in generating allelic polymorphism at an $\mathrm{MHC}$ class II locus in Peromyscus maniculatus. Genetics Research 82:89.99

Sargis E (2004) New views on tree shrews: the role of tupaiids in primate supraordinal relationships. Evol Anthropol 13:56-66 
Sato A, Figueroa F, O'hUigin C, Steck N, Klein J (1998) Cloning of major histocompatibility complex (Mhc) genes from threespine stickleback, Gasterosteus aculeatus. Mol Mar Biol Biotechnol 7:221-231

Sawai H, Go Y, Satta Y (2008) Biological implication for loss of function at major histocompatibility complex loci. Immunogenetics 60:295-302

Sawyer SA (1999) GENECONV: a computer package for the statistical detection of gene conversion. Distributed by the author, Department of Mathematics, Washington University, St. Louis

Schad J, Sommer S, Ganzhorn JU (2004) MHC variability of a small lemur in the littoral forest fragments of southeastern Madagascar. Conserv Genet 5:299-309

Schaschl H, Suchentrunk F, Hammer S, Goodman SJ (2005) Recombination and the origin of sequence diversity in the DRB MHC class II locus in chamois (Rupicapra spp.). Immunogenetics $57: 108-115$

Sittisombut N, Tissof RG, Knight KL (1989) Rabbit major histocompatibility complex III. Multiple class II DRß genes and restriction frament length polymorphism of the class II $\alpha$ and $\beta$ genes. Int $J$ Immunogenet 16:63-75

Sommer S (2005) The importance of immune gene variability (MHC) in evolutionary ecology and conservation. Frontiers in Zoology 2:16

Stoffels RJ, Spencer HG (2008) An asymmetric model of heterozygote advantage at major histocompatibility complex genes: degenerate pathogen recognition and intersection advantage. Genetics 178:1473-1489

Tamura K, Dudley J, Nei M, Kumar S (2007) MEGA4: molecular evolutionary genetics analysis (MEGA) software version 4.0. Mol Biol Evol 24:1596-1599

The MHC sequencing consortium (1999) Complete sequence and gene map of a human major histocompatibility complex. Nature $401: 921-923$

Trowsdale J (1995) "Both man \& bird \& beast": comparative organization of MHC genes. Immunogenetics 41:1-17

Van Den Bussche RA, Hoofer SR, Lochmiller RL (1999) Characterization of Mhc-DRB allelic diversity in white-tailed deer (Odocoileus virginianus) provides insight into Mhc-DRB allelic evolution within Cervidae. Immunogenetics 49:429-437

van Oosterhout C (2009) A new theory of MHC evolution: beyond selection on the immune genes. Proceedings of the Royal Society B: Biological Sciences 276:657-665

Wegner KM, Kalbe M, Schaschl H, Reusch TBH (2004) Parasites and individual major histocompatibility complex diversity - an optimal choice? Microbes Infect 6:1110-1116

Westerdahl H (2007) Passerine MHC: genetic variation and disease resistance in the wild. J Ornithol 148:469-477

Yeager M, Hughes AL (1999) Evolution of the mammalian MHC: natural selection, recombination, and convergent evolution. Immunol Rev 167:45-58 